\title{
Cytosolic Iron-Sulfur Protein Assembly 1 (CIAO1) Downstream Activation of Phospholipase A2 and Hormone-Mediated Signaling-Induced Cell Death Network in Human Hepatocellular Carcinoma (HCC) by Systems- Theoretical Analysis
}

\author{
Lianxiu Qi ${ }^{1}$, Lin Wang ${ }^{1 *}$, Minghu Jiang ${ }^{2}$, Juxiang Huang ${ }^{1}$ and Hong Lin ${ }^{1}$ \\ ${ }^{1}$ Biomedical Center, School of Electronic Engineering, Beijing University of Posts and Telecommunications, Beijing, 100876, China \\ ${ }^{2}$ Lab of Computational Linguistics, School of Humanities and Social Sciences, Tsinghua University, Beijing, 100084, China
}

\begin{abstract}
We constructed the significant high expression (fold change $\geq 2$ ) cytosolic iron-sulfur protein assembly 1 (CIAO1) downstream activation of phospholipase A2 and hormone-mediated signaling-induced cell death network in human Hepato Cellular Carcinoma (HCC), compared with low expression no-tumor hepatitis/cirrhotic tissues (HBV or HCV infection) in GEO data set, by using integration of gene regulatory activated and inhibited network inference method. Our result showed that $\mathrm{CIAO1}$ downstream activation of phospholipase $\mathrm{A} 2$ and hormone-mediated signaling-induced cell death upstream network had no result, and downstream CIAO1-activated PLA2G1B, NUP62 in HCC. By integrative analysis of biological processes simultaneous occurrence between the different CIAO1 activated downstream cell death gene ontology (GO) network of HCC compared with CIAO1 activated downstream cell death GO network of no-tumor hepatitis/cirrhotic tissues, and the same compared CIAO1 inhibited downstream cell death GO network of no-tumor hepatitis/cirrhotic tissues, or the different compared CIAO1 inhibited downstream cell death GO network of $\mathrm{HCC}$, we proposed and verified that CIAO1 activated upstream network had no result; Downstream network consisted of activation of phospholipase $\mathrm{A} 2$, cell death, protein kinase cascade, regulation of signal transduction, hormonemediated signaling, negative regulation of epidermal growth factor receptor signaling pathway, negative regulation of MAPK (Mitogen Activated Protein Kinase) activity, negative regulation of Ras protein signal transduction in HCC, as a result of downstream activation of phospholipase A2 and hormone-mediated signaling-induced cell death in HCC.
\end{abstract}

Keywords: Cytosolic iron-sulfur protein assembly 1 (CIAO1); Human hepatocellular carcinoma (HCC); Downstream activation of phospholipase A2 and hormone-mediated signaling-induced cell death network; Systems-theoretical analysis

\section{Introduction}

Cytosolic iron-sulfur protein assembly 1 (CIAO1) is our identified significant high expression (fold change $\geq 2$ ) gene in human hepatocellular carcinoma (HCC) compared with low expression notumor hepatitis/cirrhotic tissues (HBV or HCV infection) from GEO (Gene Expression Omnibus) data set GSE10140-10141(http://www. ncbi.nlm.nih.gov/geo/query/acc.cgi?acc=GSE10140, http://www.ncbi. nlm.nih.gov/geo/query/acc.cgi?acc=GSE10141) [1].

We interpreted PLA2G1B and NUP62 by using gene ontology (GO). PLA2G1B cellular component, molecular function and biological process are relevant to extracellular region, extracellular space; receptor binding, calcium ion binding, hydrolase activity, bile acid binding, cell surface binding, calcium-dependent phospholipase A2 activity; activation of MAPK activity, neutrophil mediated immunity, fatty acid biosynthesis, phospholipid metabolism, actin filament organization, signal transduction, protein kinase cascade, glucose transport, leukotriene biosynthesis, neutrophil chemotaxis, organismal lipid catabolism, positive regulation of DNA replication, phosphatidylcholine metabolism, positive regulation of fibroblast proliferation, arachidonic acid secretion, positive regulation of protein secretion, positive regulation of immune response, activation of NFkappaB transcription factor, positive regulation of calcium ion transport into cytosol, positive regulation of specific transcription from RNA polymerase II promoter, activation of phospholipase A2, interleukin-8 production, cellular response to insulin stimulus (GO database). NUP62 cellular component, molecular function and biological process are relevant to nucleus, nuclear pore, nucleolus, cytoplasm, centrosome, nucleocytoplasmic shuttling complex, nuclear membrane; chromatin binding, protein serine/threonine kinase activity, nucleocytoplasmic transporter activity, protein binding, structural constituent of nuclear pore, receptor signaling complex scaffold activity, transcription regulator activity, $\mathrm{SH} 2$ domain binding, ubiquitin binding, thyroid hormone receptor binding, PTB (Phosphotyrosine-binding) domain binding; cell surface receptor linked signal transduction, cell aging, cell death, negative regulation of cell proliferation, hormone-mediated signaling, regulation of signal transduction, protein transport, negative regulation of epidermal growth factor receptor signaling pathway, negative regulation of apoptosis, positive regulation of I-kappaB kinase/NF-kappaB cascade, negative regulation of MAPK activity, positive regulation of epidermal growth factor receptor signaling pathway, positive regulation of transcription, negative regulation of Ras protein signal transduction, mRNA transport, intracellular protein transmembrane transport (GO database).

Study of cytosolic iron-sulfur protein assembly 1 (CIAO1) is presented in several papers as follows: Mouse knock-out of IOP1 protein reveals its indispensable role in mammalian cytosolic iron-sulfur protein biogenesis [2]; Tah18 transfers electrons to Dre2 in cytosolic iron-sulfur protein biogenesis [3]; A role for IOP1 in mammalian

*Corresponding author: Lin Wang, Biomedical Center, School of Electronics Engineering, Beijing University of Posts and Telecommunications, Beijing, 100876, China Tel: 0086-13240981826; Fax: 8610-62785736; E-mail: wanglin98@tsinghua.org.cn

Received March 26, 2012; Accepted April 21, 2012; Published April 27, 2012

Citation: Qi L, Wang L, Jiang M, Huang J, Lin H (2012) Cytosolic Iron-Sulfur Protein Assembly 1 (CIAO1) Downstream Activation of Phospholipase A2 and Hormone-Mediated Signaling-Induced Cell Death Network in Human Hepatocellular Carcinoma (HCC) by Systems-Theoretical Analysis. Mol Biol 1:105. doi:10.4172/2168-9547.1000105

Copyright: @ 2012 Qi L, et al. This is an open-access article distributed under the terms of the Creative Commons Attribution License, which permits unrestricted use, distribution, and reproduction in any medium, provided the original author and source are credited. 
cytosolic iron-sulfur protein biogenesis [4]; The essential cytosolic ironsulfur protein $\mathrm{Nbp} 35$ acts without Cfd1 partner in the green lineage [5]; Role of human mitochondrial Nfs1 in cytosolic iron-sulfur protein biogenesis and iron regulation [6]; Activation of the iron regulon by the yeast Aft1/Aft2 transcription factors relys on mitochondrial but not cytosolic iron-sulfur protein biogenesis [7]; Gene structure and mutation causing X-linked sideroblastic anemia with ataxia with cytosolic iron-sulfur protein maturation disruption [8]. Downstream activation of phospholipase A2 and hormone-mediated signalinginduced cell death is presented in some papers. Such as, Cytoplasmic phospholipase A2 levels correlate with apoptosis in human colon tumorigenesis [9]; Potentiation by vitamin D analogs of TNFalpha and ceramide-induced apoptosis in MCF-7 cells is related to activation of cytosolic phospholipase A2 [10]; 1,25-Dihydroxyvitamin D3 protects human leukemic cells from tumor necrosis factor-induced apoptosis through inactivation of cytosolic phospholipase A2 [11]; Phospholipase A2-activating protein (PLAA) enhances cisplatin-induced apoptosis in HeLa cells [12]; Alpha-Tocopheryl succinate contributes to apoptosis in erbB2-expressing breast cancer cell via NF-kappaB pathway [13]; Gene network signaling in hormone responsiveness modifies autophagy and apoptosis in breast cancer cells [14]; Rho/ROCK/actin signaling regulates membrane androgen receptor induced apoptosis in prostate cancer cells [15]; Gonadotropin-releasing hormone type II contributes to apoptosis of human endometrial cancer cells by activating GADD45alpha [16]. Yet the distinct high expression CIAO1 downstream activation of phospholipase A2 and hormone-mediated signaling-induced cell death network in HCC remains to be elucidated. Here we constructed the high expression CIAO1 activated downstream activation of phospholipase A2 and hormone-mediated signalinginduced cell death network in HCC from GEO data set by gene regulatory network inference method based on linear programming and decomposition procedure.

In this study, we constructed CIAO1 upstream and downstream activated and inhibited downstream activation of phospholipase A2 and hormone-mediated signaling-induced cell death network in no-tumor hepatitis/cirrhotic tissues and HCC. The biological process and data analysis of the low- and high- expression CIAO1 downstream activation of phospholipase A2 and hormone-mediated signaling-induced cell death network was done in no-tumor hepatitis/cirrhotic tissues (HBV or HCV infection) and HCC by gene ontology (GO) database. By comparison with the same and different upstream and downstream GO and numbers of CIAO1 activated and inhibited downstream cell death network between no-tumor hepatitis/cirrhotic tissues and HCC, we put forward hypothesis of CIAO1 activated downstream cell death network of downstream activation of phospholipase A2 and hormonemediated signaling-induced cell death in HCC.

\section{Materials and Methods}

Microarrays 6,144 genes were used for analyzing CIAO1 downstream cell death mechanism and constructing molecular network of HCC from our total network of 225 significant high expression (fold change $\geq 2$ ) molecules in HCC compared with no-tumor hepatitis/cirrhotic tissues (HBV or HCV infection) based on GEO data set GSE10140-10141 (http://www.ncbi.nlm.nih.gov/geo/query/ acc.cgi?acc=GSE10140, http://www.ncbi.nlm.nih.gov/geo/query/acc. cgi?acc $=$ GSE10141). The raw microarray data was preprocessed by log base 2 .

Significant expressed genes for studying CIAO1 downstream cell death mechanism and molecular network were identified using significant analysis of microarrays (SAM) (http://www-stat.stanford. edu/ tibs/SAM/) [17]. We selected two classes unpaired and minimum fold change $\geq 2$ and chose the significant highly expressed value genes of HCC compared with that of no-tumor hepatitis/cirrhotic tissues (HBV or HCV infection) under the false-discovery rate was $0 \%$.

CIAO1 downstream activation of phospholipase A2 and hormonemediated signaling-induced cell death network was constructed based on GRNInfer and GVedit tools (http://www.graphviz.org/ About.php). GRNInfer is a novel mathematic method called GNR (Gene Network Reconstruction tool) based on linear programming and a decomposition procedure for inferring gene network [18]. We established CIAO1 activated network of HCC based on the fold change $\geq 2$ distinguished genes and selected parameters as lambda 0.0 because we used one data set. Lambda was a positive parameter which balanced the matching and sparsity terms in the objective function. Using different thresholds, we could predict various networks with the different edge density. The threshold parameters make the edge whose strength of link is smaller than threshold not shown in the network graph. The smaller this parameter, the more edges in the network graph. We selected threshold as 1.0e-7.

CIAO1 downstream cell death mechanism of HCC was analyzed using Molecule Annotation System, MAS (CapitalBio Corporation, Beijing, China; http://bioinfo.capitalbio.com/mas3/). MAS is a Webbased software toolkit for a whole data mining and function annotation solution to extract and analyze biological molecules relationships from public databases. The primary databases of MAS integrated various well-known biological resources, such as Gene Ontology (http://www. geneontology.org), KEGG (http://www.genome.jp/kegg/), BioCarta (http://www.biocarta.com/), GenMapp (http://www.genmapp.org/), HPRD (http://www.hprd.org/), MINT (http://mint.bio.uniroma2.it/ mint/Welcome.do), BIND (http://www.blueprint.org/), Intact (http:// www.ebi.ac.uk/intact/), UniGene (www.ncbi.nlm.nih.gov/UniGen), OMIM (http://www.ncbi.nlm.nih.gov/sites/entrez? $\mathrm{db}=\mathrm{omim}$ ) and disease ((http://bioinfo.capitalbio.com/mas3/). MAS offers various query entries and graphics. The algorithm is $\mathrm{P}, \mathrm{Q}$ value in $\mathrm{GO}$ and pathway of module was presented in reference [19].

\section{Results}

We analyzed CIAO1 downstream cell death mechanism and constructed molecular network of HCC from our total network of 225 significant high expression molecules (fold change $\geq 2$ ) from 6,144 genes of HCC compared with no-tumor hepatitis/cirrhotic tissues (HBV or HCV infection) by GRNInfer, respectively.

We extracted the biological process of GO terms and did numbers data analysis of the different biological processes of CIAO1 activated downstream cell death network in HCC compared with activated network of no-tumor hepatitis/cirrhotic tissues, the same biological processes of CIAO1 activated downstream cell death network in HCC compared with inhibited network of no-tumor hepatitis/cirrhotic tissues, as shown in Table 1. GO terms and numbers data was analyzed the different biological processes of CIAO1 activated compared with inhibited downstream cell death network in HCC, as shown in Table 2.

CIAO1 activated downstream activation of phospholipase A2 and hormone-mediated signaling-induced cell death network was constructed in HCC. Our result showed that upstream had no result, and the downstream CIAO1-activated PLA2G1B, NUP62 in HCC, as shown in Figure 1 and Figure 2.

\section{Discussion}

Our aim is to study novel high expression CIAO1 downstream 
Citation: Qi L, Wang L, Jiang M, Huang J, Lin H (2012) Cytosolic Iron-Sulfur Protein Assembly 1 (CIAO1) Downstream Activation of Phospholipase A2 and Hormone-Mediated Signaling-Induced Cell Death Network in Human Hepatocellular Carcinoma (HCC) by Systems-Theoretical Analysis. Mol Biol 1:105. doi:10.4172/2168-9547.1000105

Page 3 of 5

\begin{tabular}{|c|c|}
\hline \multicolumn{2}{|c|}{$\begin{array}{l}\text { The Different Biological Processes of CIAO1 Activated Downstream Cell Death Network of HCC Compared with Activated Network of No-tumor Hepatitis/ } \\
\text { cirrhotic Tissues }\end{array}$} \\
\hline Terms & Numbers \\
\hline protein kinase cascade & 1 \\
\hline activation of phospholipase A2 & 1 \\
\hline regulation of signal transduction & 1 \\
\hline cell death & 1 \\
\hline hormone-mediated signaling & 1 \\
\hline negative regulation of epidermal growth factor receptor signaling pathway & 1 \\
\hline negative regulation of MAPK activity & 1 \\
\hline negative regulation of Ras protein signal transduction & 1 \\
\hline fatty acid biosynthesis & 1 \\
\hline organismal lipid catabolism & 1 \\
\hline phosphatidylcholine metabolism & 1 \\
\hline phospholipid metabolism & 1 \\
\hline \multicolumn{2}{|c|}{$\begin{array}{l}\text { The Same Biological Processes of CIAO1 Activated Downstream Cell Death Network of HCC Compared with Inhibited Network of No-tumor Hepatitis/ } \\
\text { cirrhotic Tissues }\end{array}$} \\
\hline Terms & Numbers \\
\hline None & 0 \\
\hline
\end{tabular}

Table 1: GO Terms and numbers data analysis of the different biological processes of CIAO1 activated downstream cell death network of HCC compared with activated network of no-tumor hepatitis/cirrhotic tissues, the same biological processes of CIAO1 activated downstream cell death network of HCC compared with inhibited network of no-tumor hepatitis/cirrhotic tissues.

\begin{tabular}{|l|l|}
\hline \multicolumn{1}{|c|}{ The Different Biological Processes of CIAO1Activated Downstream Cell Death Network of HCC Compared with Inhibited Network of HCC } \\
\hline \multicolumn{1}{|c|}{ Terms } \\
\hline negative regulation of epidermal growth factor receptor signaling pathway \\
\hline negative regulation of MAPK activity & 1 \\
\hline negative regulation of Ras protein signal transduction & 1 \\
\hline protein kinase cascade & 1 \\
\hline regulation of signal transduction & 1 \\
\hline activation of phospholipase A2 & 1 \\
\hline cell death & 1 \\
\hline hormone-mediated signaling & 1 \\
\hline fatty acid biosynthesis & 1 \\
\hline organismal lipid catabolism & 1 \\
\hline phosphatidylcholine metabolism & 1 \\
\hline phospholipid metabolism & 1 \\
\hline
\end{tabular}

Table 2: GO Terms and numbers data analysis of the different biological processes of CIAO1 activated downstream cell death network of HCC compared with inhibited network of HCC.

cell death mechanism and molecular network in HCC. We have already constructed and analyzed some novel molecular network from different databases presented in our articles [19-32]. In this study, we constructed CIAO1 upstream and downstream activated and inhibited downstream activation of phospholipase A2 and hormone-mediated signaling-induced cell death network in no-tumor hepatitis/cirrhotic tissues and HCC. The biological process and data analysis of the lowand high expression CIAO1 downstream activation of phospholipase A2 and hormone-mediated signaling-induced cell death network was done in no-tumor hepatitis/cirrhotic tissues (HBV or HCV infection) and HCC by GO database. By comparison with the same and different upstream and downstream GO and numbers of CIAO1 activated and inhibited downstream cell death network between no-tumor hepatitis/ cirrhotic tissues and HCC, we put forwards hypothesis of CIAO1 activated downstream cell death network of downstream activation of phospholipase A2 and hormone-mediated signaling-induced cell death in HCC.

We extracted the biological process of GO terms and did numbers data analysis of the different biological processes of CIAO1 activated downstream cell death network in HCC compared with activated network of no-tumor hepatitis/cirrhotic tissues, the same biological processes of CIAO1 activated downstream cell death network in HCC compared with inhibited network of no-tumor hepatitis/cirrhotic tissues (Table 1 and Table 2). We constructed the high expression (fold change $\geq 2$ ) CIAO1 activated downstream activation of phospholipase A2 and hormone-mediated signaling-induced cell death network in human hepatocellular carcinoma (HCC) compared with low expression no-tumor hepatitis/cirrhotic tissues (HBV or HCV infection) in GEO data set using integration of gene regulatory network inference method. Our result showed that CIAO1 downstream activation of phospholipase A2 and hormone-mediated signaling-induced cell death upstream network had no result, and downstream CIAO1-activated PLA2G1B, NUP62 (Figure 1 and Figure 2) in HCC.

By further comparison with the same biological processes of and different gene ontology (GO) of CIAO1 activated and inhibited downstream cell death network between no-tumor hepatitis/cirrhotic tissues and HCC, we found that the different biological processes of CIAO1 activated upstream network had no result; Downstream network consisted of protein kinase cascade, activation of phospholipase A2, regulation of signal transduction, cell death, hormone-mediated signaling, negative regulation of epidermal growth factor receptor signaling pathway, negative regulation of MAPK activity, negative regulation of Ras protein signal transduction in HCC compared with activated network of no-tumor hepatitis/cirrhotic tissues. 
Citation: Qi L, Wang L, Jiang M, Huang J, Lin H (2012) Cytosolic Iron-Sulfur Protein Assembly 1 (CIAO1) Downstream Activation of Phospholipase A2 and Hormone-Mediated Signaling-Induced Cell Death Network in Human Hepatocellular Carcinoma (HCC) by Systems-Theoretical Analysis. Mol Biol 1:105. doi:10.4172/2168-9547.1000105

Page 4 of 5

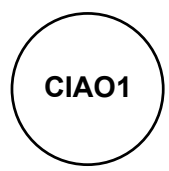

Figure 1: CIAO1 upstream activated phospholipase A2 and hormonemediated signaling-induced cell death network in HCC by GRNInfer and our programming.

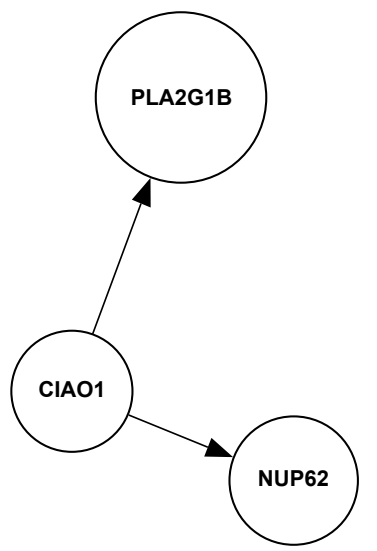

Figure 2: CIAO1 downstream activated phospholipase $A 2$ and hormonemediated signaling-induced cell death network in HCC by GRNInfer and our programming

The same biological processes of CIAO1 activated upstream network had no result; downstream network had no result in HCC compared with inhibited network of no-tumor hepatitis/cirrhotic tissues.

The different biological processes of CIAO1 activated network had no result; Downstream network included negative regulation of epidermal growth factor receptor signaling pathway, negative regulation of MAPK activity, negative regulation of Ras protein signal transduction, protein kinase cascade, regulation of signal transduction, activation of phospholipase A2, cell death, hormone-mediated signaling compared with inhibited network of HCC.

By integrative analysis of biological processes simultaneous occurrence between the different CIAO1 activated downstream cell death gene ontology (GO) network of HCC compared with CIAO1 activated downstream cell death GO network of no-tumor hepatitis/ cirrhotic tissues, and the same compared CIAO1 inhibited downstream cell death GO network of no-tumor hepatitis/cirrhotic tissues, or the different compared CIAO1 inhibited downstream cell death GO network of HCC, we proposed and verified that CIAO1 activated upstream network had no result; Downstream network included activation of phospholipase A2, cell death, protein kinase cascade, regulation of signal transduction, hormone-mediated signaling, negative regulation of epidermal growth factor receptor signaling pathway, negative regulation of MAPK activity, negative regulation of Ras protein signal transduction in HCC, as a result of downstream activation of phospholipase A2 and hormone-mediated signalinginduced cell death in HCC.

\section{Acknowledgments}

This work was supported by the National Natural Science Foundation of China (No.61171114), the Returned Overseas Chinese Scholars for Scientific research Foundation of State Education Ministry, Significant Science and Technology
Project for New Transgenic Biological Species (2009ZX08012-001B), Automatica Scientific Planning of Tsinghua University (20111081023 and 20111081010), State Key Lab of Pattern Recognition Open Foundation.

\section{References}

1. Hoshida Y, Villanueva A, Kobayashi M, Peix J, Chiang DY, et al. (2008) Gene expression in fixed tissues and outcome in hepatocellular carcinoma. $\mathrm{N}$ Engl $J$ Med 359: 1995-2004

2. Song D, Lee FS (2012) Mouse knock-out of IOP1 protein reveals its essentia role in mammalian cytosolic iron-sulfur protein biogenesis. J Biol Chem 286 : 15797-15805

3. Netz DJ, Stumpfig M, Dore C, Muhlenhoff U, Pierik AJ, et al. (2012) Tah18 transfers electrons to Dre2 in cytosolic iron-sulfur protein biogenesis. Nat Chem Biol 6: 758-765.

4. Song D, Lee FS (2008) A role for IOP1 in mammalian cytosolic iron-sulfur protein biogenesis. J Biol Chem 283: 9231-9238.

5. Bych K, Netz DJ, Vigani G, Bill E, Lill R, et al. (2008) The essential cytosolic iron-sulfur protein $\mathrm{Nbp} 35$ acts without $\mathrm{Cfd} 1$ partner in the green lineage. J Biol Chem 283: 35797-35804.

6. Biederbick A, Stehling O, Rosser R, Niggemeyer B, Nakai Y, et al. (2006) Role of human mitochondrial Nfs1 in cytosolic iron-sulfur protein biogenesis and iron regulation. Mol Cell Biol 26: 5675-5687.

7. Rutherford JC, Oieda L, Balk J, Muhlenhoff U, Lill R, et al. (2005) Activation of the iron regulon by the yeast Aft $1 /$ Aft2 transcription factors depends on mitochondrial but not cytosolic iron-sulfur protein biogenesis. J Biol Chem 280 10135-10140.

8. Bekri S, Kispal G, Lange H, Fitzsimons E, Tolmie J, et al. (2000) Human ABC7 transporter: gene structure and mutation causing $X$-linked sideroblastic anemia with ataxia with disruption of cytosolic iron-sulfur protein maturation. Blood 96 3256-3264.

9. Dong M, Johnson M, Rezaie A, Ilsley JN, Nakanishi M, et al. (2005) Cytoplasmic phospholipase A2 levels correlate with apoptosis in human colon tumorigenesis. Clin Cancer Res 11: 2265-2271.

10. Pirianov G, Danielsson C, Carlberg C, James SY, Colston KW (1999) Potentiation by vitamin D analogs of TNFalpha and ceramide-induced apoptosis in MCF-7 cells is associated with activation of cytosolic phospholipase A2. Cel Death Differ 6: 890-901.

11. Wu YL, Jiang XR, Lillington DM, Allen PD, Newland AC, et al. (1998) 1,25-Dihydroxyvitamin D3 protects human leukemic cells from tumor necrosis factor-induced apoptosis via inactivation of cytosolic phospholipase A2. Cancer Res 58: 633-640.

12. Zhang F, Suarez G, Sha J, Sierra JC, Peterson JW, et al. (2009) Phospholipase A2-activating protein (PLAA) enhances cisplatin-induced apoptosis in HeLa cells. Cell Signal 21: 1085-1099.

13. Wang XF, Xie Y, Wang HG, Zhang Y, Duan XC, et al. (2010) a-Tocophery succinate induces apoptosis in erbB2-expressing breast cancer cell via NF- $\mathrm{B}$ pathway. Acta Pharmacol Sin 31: 1604-1610.

14. Clarke R, Shahjahan AN, Riggins RB, Cho Y, Crawford A, et al. (2009) Gene network signaling in hormone responsiveness modifies apoptosis and autophagy in breast cancer cells. J Steroid Biochem Mol Biol 114: 8-20.

15. Papadopoulou N, Charalampopoulos I, Alevizopoulos K, Gravanis A Stournaras C, et al. (2008) Rho/ROCK/actin signaling regulates membrane androgen receptor induced apoptosis in prostate cancer cells. Exp Cell Res 314: 3162-3174

16. Wu HM, Cheng JC, Wang HS, Huang HY, MacCalman CD, et al. (2009) Gonadotropin-releasing hormone type II induces apoptosis of human endometrial cancer cells by activating GADD45alpha. Cancer Res 69: 4202 4208.

17. Storey JD (2002) A direct approach to false discovery rates. J R Stat Soc Series B Stat Methodol 64: 479-498.

18. Wang $Y$, Joshi T, Zhang XS, Xu D, Chen L (2006) Inferring gene regulatory networks from multiple microarray datasets. Bioinformatics 22: 2413-2420.

19. Wang L, Sun L, Huang J, Jiang M (2011) Cyclin-dependent kinase inhibito 3 (CDKN3) novel cell cycle computational network between human nonmalignancy associated hepatitis/cirrhosis and hepatocellular carcinoma (HCC) transformation. Cell Prolif 44: 291-299. 
Citation: Qi L, Wang L, Jiang M, Huang J, Lin H (2012) Cytosolic Iron-Sulfur Protein Assembly 1 (CIAO1) Downstream Activation of Phospholipase A2 and Hormone-Mediated Signaling-Induced Cell Death Network in Human Hepatocellular Carcinoma (HCC) by Systems-Theoretical Analysis. Mol Biol 1:105. doi:10.4172/2168-9547.1000105

Page 5 of 5

20. Wang L, Sun Y, Jiang M, Zheng X (2009) Integrative decomposition procedure and Kappa statistics for the distinguished single molecular network construction and analysis. J Biomed Biotechnol 2009: 726728

21. Wang L, Sun Y, Jiang M, Zhang S, Wolfl S (2009) FOS proliferating network construction in early colorectal cancer (CRC) based on integrative significant function cluster and inferring analysis. Cancer Invest 27: 816-824.

22. Wang L, Huang J, Jiang M, Zheng X (2010) AFP computational secreted network construction and analysis between human hepatocellular carcinoma (HCC) and no-tumor hepatitis/cirrhotic liver tissues. Tumour Biol 31: 417-425.

23. Wang L, Huang J, Jiang M, Sun L (2011) MYBPC1 computational phosphoprotein network construction and analysis between frontal cortex of HIV encephalitis (HIVE) and HIVE-control patients. Cell Mol Neurobiol 31: 233 241

24. Wang L, Huang J, Jiang M, Sun L (2011) Survivin (BIRC5) cell cycle computational network in human no-tumor hepatitis/cirrhosis and hepatocellular carcinoma transformation. J Cell Biochem 112: 1286-1294.

25. Wang L, Huang J, Jiang M, Lin H (2012) Signal Transducer and Activator of Transcription 2 (STAT2) Metabolism Coupling Postmitotic Outgrowth to Visual and Sound Perception Network in Human Left Cerebrum by Biocomputation. $\mathrm{J}$ Mol Neurosci.

26. Wang L, Huang J, Jiang M (2010) RRM2 computational phosphoprotein network construction and analysis between no-tumor hepatitis/cirrhotic liver tissues and human hepatocellular carcinoma (HCC). Cell Physiol Biochem 26: 303-310.
27. 27 ) Wang, L, Huang J, Jiang M (2011) CREB5 computational regulation network construction and analysis between frontal cortex of HIV encephalitis (HIVE) and HIVE-control patients. Cell Biochem Biophys 60: 199-207.

28. Sun Y, Wang L, Liu L (2008) Integrative decomposition procedure and Kappa statistics set up ATF2 ion binding module in Malignant Pleural Mesothelioma (MPM). Frontiers of Electrical and Electronic Engineering in China 3: 381-387.

29. Sun Y, Wang L, Jiang M, Huang J, Liu Z, et al. (2010) Secreted Phosphoprotein 1 Upstream Invasive Network Construction and Analysis of Lung Adenocarcinoma Compared with Human Normal Adjacent Tissues by Integrative Biocomputation. Cell Biochem Biophys 56: 59-71.

30. Sun L, Wang L, Jiang M, Huang J, Lin H (2011) Glycogen debranching enzyme 6 (AGL), enolase 1 (ENOSF1), ectonucleotide pyrophosphatase 2 (ENPP2_1) glutathione S-transferase 3 (GSTM3_3) and mannosidase (MAN2B2) metabolism computational network analysis between chimpanzee and human left cerebrum. Cell Biochem Biophys 61: 493-505.

31. Huang JX, Wang L, Jiang MH (2010) TNFRSF11B computational development network construction and analysis between frontal cortex of HIV encephalitis (HIVE) and HIVE-control patients. J Inflamm (Lond) 7: 50.

32. Huang J, Wang L, Jiang M, Zheng X (2010) Interferon a-Inducible Protein 27 Computational Network Construction and Comparison between the Frontal Cortex of HIV Encephalitis (HIVE) and HIVE-Control Patients. The Open Genomics Journal 3: 1-8. 